\title{
L'école et la justice sociale. Une étude de cas autour de No Child Left Behind
}

\author{
Georges Felouzis, Université de Genève \\ Mélanie Savioz, Université de Genève
}

DOI : 10.51186/journals/ed.2021.11-1.e394

\section{Résumé}

Cet article est fondé sur une revue de la littérature concernant la loi américaine No Child Left Behind (NCLB). À partir de l'étude de cette loi, de son implantation et de ses effets sur l'équité et les performances scolaires, cet article vise à comprendre dans quelle mesure une politique d'équité parvient - ou pas - à limiter les inégalités scolaires. Après avoir décrit les instruments mobilisés par NCLB, nous explicitons le rôle de sa mise en œuvre dans la répartition des biens éducatifs, en nous demandant quels principes institutionnels organisent cette répartition et en mettant en lumière les mécanismes de découplage entre les intentions de NCLB et leur réalisation. II ressort de nos analyses que la loi présente des effets pervers: les écoles pauvres et ethniquement diverses ont plus de probabilités de ne pas respecter les exigences de NCLB et d'en être sanctionnées, notamment financièrement. Ainsi, le principe de "pressure without support » pénalise les plus défavorisés. In fine, la thèse défendue dans cet article est qu'une politique éducative de justice sociale ne peut se réaliser que si elle se fonde sur une vision juste des sources des inégalités et si elle considère de façon équitable l'ensemble des acteurs des processus éducatifs.

Mots-clés : découplage, égalité des chances, No Child Left Behind, politiques éducatives, reddition des comptes

\begin{abstract}
This article is based on a review of the literature on the American law No Child Left Behind (NCLB). By studying this law, its implementation and its effects on equity and school performance, this article aims to understand the extent to which an equity policy succeeds or fails - in limiting school inequalities. After describing the instruments mobilized by NCLB, we explain the role of its implementation in the distribution of educational goods, asking what institutional principles organize this distribution and highlighting the mechanisms of decoupling between the intentions of NCLB and their realization. Our analysis shows that the law has perverse effects: poor and ethnically diverse schools are more likely to fail to comply with NCLB requirements and to be penalized, especially financially. Thus, the principle of
\end{abstract}


"pressure without support" penalizes the most disadvantaged. Ultimately, the thesis supported in this article is that an educational policy of social justice can only be achieved if it is based on a fair vision of the sources of inequalities and if it considers all the actors in the educational process in an equitable manner.

Keywords: accountability, educational policies, equality of opportunity, loose coupling, No Child Left Behind

À quelles conditions l'école peut-elle être juste et équitable ? Suffit-il de garantir une égalité formelle entre les élèves, quelles que soient leurs caractéristiques, pour être quitte de la question des inégalités et de la reproduction sociale par l'école ? Ou faut-il aller plus loin en considérant qu'une école juste doit viser, voire garantir, une égalité réelle entre élèves, c'està-dire une équité de résultats, sur les plans des acquis scolaires, des parcours de formation ou encore des orientations ?

Cette alternative, que l'on pourrait résumer par le débat entre principe d'égalité d'une part et principe d'équité de l'autre (Crahay, 2012), a des conséquences fortes sur la nature de l'action publique à mettre en œuvre en éducation. Le principe d'égalité - chacun-e étant l'égal-e de l'autre en droit - implique de garantir un égal accès de toutes et tous à l'enseignement en privilégiant notamment des politiques structurelles - organisation des curricula, mode de recrutement et de formation des enseignant-es, définition des plans d'étude, etc. En revanche, le principe d'équité part du constat de l'incomplétude de l'approche par l'égalité et suscite des politiques qui visent des populations spécifiques en fonction de difficultés d'apprentissage, d'une origine sociale défavorisée, de l'appartenance à un groupe minoritaire défini selon le sexe ou l'origine ethno-raciale, ou encore d'une combinaison de ces différents facteurs. II s'agit alors de compenser les inégalités de départ - fruits de conditions sociales et économiques défavorisées par exemple, ou de discriminations envers des groupes minoritaires - en mettant en œuvre une action publique ciblée au bénéfice de populations spécifiques. Ce "principe de différence » (Rawls, 1987) consiste ainsi en la mise en œuvre d'inégalités considérées comme justes, dès lors qu'elles sont au bénéfice des plus faibles. On part, par exemple, de l'idée que l'égalité formelle entre les noir-es et les blanc-hes n'implique en rien une absence de discrimination raciale dans la vie sociale en général, ni au sein de l'école en particulier (Barton \& Coley, 2010 ; Hanushek \& Rivkin, 2006 ; Jencks, 1979), de même que l'égalité de droit selon l'origine sociale n'implique nullement une égalité de fait dans les processus d'apprentissage entre élèves de milieux sociaux contrastés (Felouzis, 2020 ; Lahire, 2019).

Dans cette perspective, nous traiterons dans cet article de la justice sociale en éducation selon un principe distributif : comment, dans quelle mesure et par quels mécanismes l'action 
publique en éducation parvient-elle - ou non - à exercer une redistribution des biens rares que sont l'éducation et les savoirs entre les différents groupes sociaux?

La question est donc de savoir comment ces politiques d'équité parviennent, et dans quelle mesure, à limiter les inégalités scolaires et par quels mécanismes. II faut pour cela raisonner sur les pratiques concrètes des acteurs' ${ }^{1}$ au sein même de l'institution (Becker, 2013) élèves, parents, enseignant-es, chef-fes d'établissement notamment - en lien avec les routines et les cadres cognitifs et normatifs qui organisent l'institution elle-même (Palier \& Surel, 2005). Cela a pour conséquence de complexifier la question de la justice distributive des biens éducatifs, car la question centrale n'est plus de définir quels en sont les principes théoriques, mais d'identifier quelles sont les actions et pratiques, qui organisent concrètement cette distribution, et comment les orienter de façon à garantir la plus grande justice sociale possible au sein des processus éducatifs.

C'est ainsi que l'on ne peut pas juger d'une action publique dans le domaine de la justice en éducation sur ses seuls principes et intentions. D'abord, parce que, pour reprendre l'article séminal de Weick (1976), les organisations éducatives se caractérisent par un découplage fort entre les intentions politiques et leur mise en œuvre concrète. Ce découplage - loose coupling - résulte des stratégies d'adaptation ou de contournement développées par les acteurs en situation. Ensuite parce que la mise en œuvre d'une politique est toujours une réinterprétation de cette politique dans un contexte institutionnel particulier (McDonnell, 2009). Le propos de cet article sera donc d'expliciter le rôle de la mise en œuvre dans les politiques éducatives visant plus de justice sociale dans la répartition des biens éducatifs, en mettant en lumière les mécanismes de découplage entre les intentions de ces politiques et leur réalisation concrète au sein des institutions éducatives.

Dans cette perspective, la politique No Child Left Behind (NCLB) est emblématique de ce type de processus institutionnel. II s'agit d'une politique qui vise notamment la justice sociale en éducation en se centrant sur les apprentissages des élèves des minorités et de milieux défavorisés sur les plans social et économique. Objet d'une abondante littérature aux ÉtatsUnis, cette politique a été largement débattue, avant, pendant et après sa mise en place au tout début des années 2000. Nous tenterons de clarifier ces débats à partir des résultats des évaluations de NCLB. II s'agira de comprendre les transformations du système éducatif étasunien qu'elle a suscitées, le poids de sa mise en œuvre contrastée au sein des États et des établissements, et, en définitive, ses effets sur la justice sociale dans les processus d'apprentissage. La base empirique de notre analyse est constituée par les articles scientifiques traitant de l'évaluation de NCLB, tant sur le plan des principes de sa conception que de sa mise en œuvre dans les différents États et établissements scolaires, ainsi que de ses effets sur les apprentissages des élèves. Nous nous basons sur un corpus d'une

1 Le genre masculin est utilisé comme générique pour le concept sociologique acteurs au sens de Hassenteufel (2011). 
trentaine d'articles centrés sur la politique NCLB publiés entre 2003 et 2021 le plus souvent dans des revues scientifiques d'éducation, de sociologie ou de sciences politiques, ainsi que des documents institutionnels décrivant les sources et modalités d'application de cette politique.

\section{SOURCES, VISÉES ET INSTRUMENTS DE NO CHILD LEFT BEHIND}

La loi No Child Left Behind prend ses racines dans un bilan sans concession du fonctionnement du système éducatif aux États-Unis. Le rapport $A$ Nation at Risk rendu public, au début des années 1980, relevait déjà les incohérences du système et son caractère très peu efficace et profondément inégalitaire. Le sous-titre du rapport, the imperative of education reform, ne laisse par ailleurs aucun doute sur les intentions de ses concepteurs et conceptrices : il est urgent de réformer l'éducation pour éviter le pire. Et de fait, le bilan établi par le rapport est alarmant. II met le doigt sur les problèmes d'apprentissage, le niveau très faible des élèves, l'ampleur des inégalités de réussite en fonction du groupe ethno-racial, l'inégale qualité de l'éducation prodiguée dans les établissements scolaires. Pour Foucault (2017) :

La commission Gardner (du nom du président de l'Université de l'Utah qui pilotait le groupe de travail du rapport) pointait du doigt toutes les faiblesses du système, en particulier ses résultats en termes d'inégalités sociales et raciales, et surtout elle faisait de l'éducation une vraie question de politique nationale, ce qu'elle n'avait jamais vraiment été, en témoigne l'introduction tardive (1980) d'un secretary for Education dans l'exécutif américain. (p. 30)

Dans ce contexte, l'inefficacité et l'inéquité de l'éducation aux États-Unis sont devenues des problèmes publics. La loi est introduite et adoptée par le Congrès américain au printemps 2001 et signée par le président G.W. Bush le 8 janvier 2002, après que le projet ait été défendu dans les deux Chambres, mais pour des raisons différentes : les républicains voyaient en NCLB le moyen d'accroître la productivité de l'école et de l'économie tandis que les démocrates, avec le Sénateur Ted Kennedy, plaidaient en faveur de l'équité (Gamoran, 2012). Les visées de la loi sont doubles : augmenter le niveau d'apprentissage général des élèves tout en diminuant les inégalités de performance entre groupes sociaux et ethnoraciaux, entre écoles, entre districts scolaires et entre États (Foucault, 2017). Au plan politique, l'objectif de la loi est d'imposer un projet centralisateur qui remet en cause la souveraineté des États fédérés. En échange, les autorités fédérales garantissent aux États une certaine autonomie quant à leur rôle dans le secteur de l'éducation et, au corps enseignant, de retenir quelques propositions des fédérations syndicales.

Pour se diriger vers ces finalités et en finir avec le « soft racism of low expectations » (Lee, 2006, p. 5), le cadre législatif, organisationnel et financier de la politique éducative est fixé. Ses principales exigences sont déclinées en quatre grands principes, et un système 
d'incitations est prévu pour amener les acteurs à diriger leur attention vers les groupes ethniques et sociaux qui en ont le plus besoin (Meuret, 2012), ainsi que pour régir le plus uniformément possible les programmes scolaires mis en place par les États (Gamoran, 2012).

Pour présenter ces principes et définir l'action publique développée dans la loi NCLB, nous proposons une entrée par les « instruments ». Au sens de Lascoumes et Le Galès (2005), " un instrument d'action publique constitue un dispositif à la fois technique et social qui organise des rapports sociaux spécifiques entre la puissance publique et ses destinataires en fonction des représentations et des significations dont il est porteur » (p. 13). Ces instruments sont, concernant la loi NCLB, au nombre de quatre. Ils sont le fruit d'une conception néolibérale de l'action publique en éducation, mêlant un principe d'autonomie des acteurs et des institutions, à celui de leur responsabilité et de régulation par les résultats.

Le premier instrument est la normalisation. L'État fédéral, jusqu'ici peu investi dans les questions d'éducation, prend un rôle moteur avec la loi NCLB. II impose la création de normes nationales (standard-based education reform) concernant d'une part le niveau d'apprentissage à atteindre pour les élèves, et d'autre part, la qualité du personnel enseignant chargé de les instruire. En effet, la loi stipule que les élèves doivent bénéficier d'un corps enseignant « hautement qualifié » ainsi que de pédagogies et de programmes fondés sur la recherche (Porter, 2007). Ainsi, être en possession d'un Bachelor et avoir passé un test d'État dans la matière transmise est un prérequis pour enseigner une matière (Bell \& Meinelt, 2011). De plus, une remise à niveau régulière par rapport à ses connaissances est demandée (Montagutelli, 2009). L'objectif ici est double. D'une part, augmenter le niveau global d'exigence, et d'autre part, réduire les écarts de performance entre élèves blanc-hes et élèves des minorités. Le législateur postule que ces normes vont pousser le personnel enseignant à trouver de nouvelles méthodes pédagogiques et de nouveaux outils pour faire réussir leurs élèves (Gamoran, 2012).

Le deuxième instrument est centré sur l'autonomie et la responsabilité des États et des établissements scolaires. Le gouvernement fédéral fournit des fonds fédéraux - destinés à financer les programmes d'études et favoriser les apprentissages des enfants économiquement défavorisés - aux États pour qu'ils les reversent aux districts scolaires qui les distribuent à leur tour aux écoles (Bell \& Meinelt, 2011). Ce mode de financement a pour but de permettre une gestion des mesures plus souple et plus indépendante du niveau local en ce qui concerne les décisions relatives aux méthodes et à la qualité de l'enseignement, aux évaluations et aux décisions pour atteindre les objectifs. Ce cadre implique aussi une plus grande responsabilité de la part des États - puisqu'ils doivent définir les indicateurs de mesure de performance - des écoles, qui doivent non seulement gérer les financements perçus, mais aussi mettre en œuvre les évaluations annuelles, ainsi que des enseignant-es dont le salaire est proportionnel à la réussite des élèves (Montagutelli, 2009). 
Le troisième instrument est celui de l'empowerment des familles des minorités et celles défavorisées au plan socioéconomique. Cet instrument prend par exemple la forme d'un «parent guide » publié par le département de l'éducation des États-Unis (Department of Education, 2003). II permet aux familles, quels que soient leurs revenus, d'avoir accès à un choix le plus large possible d'établissements, en particulier pour les parents dont les enfants fréquentent des écoles en difficulté (Montagutelli, 2009). La promotion du choix de l'école par les parents est alors considérée - au même titre que la sélection de méthodes pédagogiques - comme une des aides les plus efficaces pour combattre les inégalités scolaires (Gamoran, 2007). Pour cette raison, il est nécessaire que les parents soient informés des résultats et des moyens en vigueur de l'État et de l'établissement pour améliorer les performances de leurs enfants et il incombe aux districts d'informer les parents de la politique de NCLB et de ses implications (Lavery, 2016). L'empowerment passe donc ici par la diffusion d'informations pertinentes sur les établissements scolaires, leurs performances et leur capacité à susciter la réussite des élèves. La maîtrise de l'information sur les écoles, structurellement plus fréquente dans les milieux sociaux les plus favorisés et diplômés (Van Zanten, 2009), est ici considérée comme un outil de mobilisation des parents défavorisés et des minorités. II s'agit d'une condition nécessaire à leur engagement dans la scolarité de leurs enfants.

Le quatrième instrument, le plus connu et probablement le plus critiqué concernant cette loi, est la reddition de compte (accountability). Ce système de régulation par les résultats comprend un ensemble d'indicateurs permettant de vérifier que chacun des groupes ethnoraciaux et socioéconomiques réalise des progrès annuels (Adequate Yearly Progress - AYP) dans l'atteinte des standards et dans la réduction des écarts de performance (Foucault, 2017). Plus précisément, les élèves ont été regroupé-es de façon suivante : les étudiant-es économiquement défavorisé-es (celles et ceux bénéficiant de repas gratuits ou à prix réduit), les étudiant-es des groupes raciaux et ethniques minoritaires (noir-es, latino-américain-es, amérindien-nes, américain-es d'origine asiatique, les primo-arrivant-es), les étudiant-es en situation de handicap (qui reçoivent des services d'éducation spéciale) et les étudiant-es ne parlant pas ou peu l'anglais (Gamoran, 2012). Le rôle des États consiste à établir des objectifs précis pour chaque année d'étude et chaque sous-groupe d'élèves, traduits en indicateurs de mesure de performance, ceci afin de s'assurer que toutes les écoles atteignent les objectifs de la loi (Bell \& Meinelt, 2011). Quant aux établissements, sur la base de ces standards, ils doivent mettre en place des évaluations normatives annuelles qui mesurent les connaissances des élèves dans les matières mentionnées. Ces AYP sont enregistrés afin d'indiquer si les élèves se dirigent vers l'objectif ultime de " compétence » fixé. À la suite de l'obtention des résultats, les écoles sont alors répertoriées en deux groupes : celles atteignant et celles n'atteignant pas cet objectif. Dans le cas où un établissement ne réaliserait pas les AYP escomptés, la loi comprend un système progressif d'interventions (Bell \& Meinelt, 2011), allant du plan d'amélioration et davantage de subventions à des dispositifs plus contraignants. Si l'école n'atteint pas les objectifs fixés deux années de suite, les élèves 
doivent avoir la possibilité de changer d'établissements et d'obtenir un soutien scolaire gratuit (Garmoran, 2012). Pour l'école de départ, cela se traduit par l'obligation d'aider à la transition, de payer les frais de transport ou d'utiliser les fonds alloués par le programme NCLB pour appuyer des services supplémentaires aux élèves, comme des séances de tutorat privé (Foucault, 2017). Foucault (2017) explique qu'au bout de quatre années consécutives d'échec, l'école est pour ainsi dire sanctionnée en étant soit prise en charge par l'État, soit convertie en Charter School2. En dernier ressort, l'école subit une réorganisation totale " allant jusqu'à la fermeture [...], au licenciement du personnel et à sa réouverture avec une nouvelle équipe » (Gamoran, 2012, p. 15).

En définitive, la loi NCLB procède d'un double mouvement apparemment contradictoire. Le premier mouvement est le renforcement de l'État fédéral qui est à la source de cette politique. II fixe les objectifs, édicte des normes et standards en matière d'apprentissage, engage des budgets, suscite le choix des familles en matière d'établissement. En ce sens, la loi renforce le rôle fédéral par rapport aux États et aux districts scolaires. Le deuxième mouvement en revanche relève d'une conception néo-libérale de la société et de l'école : la régulation se fait par les lois du marché que l'on peut résumer par une mise en concurrence des établissements dans un espace donné, via un instrument d'accountability " dur » qui implique un système de sanctions et récompenses pour les établissements en fonction de la progression de leurs élèves. Enfin, cela implique de favoriser le libre choix des parents en matière de scolarisation (Felouzis, et al., 2013).

Dans ce contexte, la question est de savoir comment cette politique, dont une des visées principales est la justice sociale en éducation, s'est effectivement réalisée au contact de l'institution éducative et des différents acteurs institutionnels impliqués dans sa mise en œuvre.

\section{COUPLAGE ET DÉCOUPLAGE AU SEIN DES INSTITUTIONS ÉDUCATIVES}

NCLB, comme toute politique éducative, ne peut se résumer à ses visées et aux instruments d'action publique qu'elle mobilise. C'est à l'aune de sa mise en œuvre ainsi qu'à ses effets réels sur la société que l'on peut véritablement qualifier l'action publique, en éducation comme dans les autres domaines de la société. Pour rendre compte du décalage entre les intentions d'une politique et son implantation, nous pouvons mobiliser le concept de découplage. Ce concept classique de la sociologie des institutions a donné lieu à plusieurs travaux dans le domaine de l'éducation (Coburn 2004 ; Dumay, et al., 2013 ; Lessard \& Carpentier, 2015 ; Weick, 1976). II permet de comprendre la façon dont les visées d'une politique sont repensées, réinterprétées, voire transformées, par les acteurs au sein des institutions concernées. Si l'on suit Weick (1976), l'éducation est le domaine dans lequel ce phénomène de découplage entre ce qui est prescrit par une politique et ce qui est

2 Les Charter Schools sont des écoles sous contrat - à financement public, mais à gestion privée ou administrée par un groupe public, plutôt que par le district scolaire, et pouvant donc s'émanciper pour une large part à la réglementation en vigueur (Gamoran, 2012). 
véritablement réalisé par les acteurs est le plus marqué. Cela s'explique par la force de l'institution éducative ainsi que par la large autonomie dont jouissent les acteurs en son sein, notamment les enseignants et enseignantes.

Dans le cas de NCLB, la littérature a pu insister sur plusieurs formes de découplage qui ont eu une influence importante sur la capacité de cette politique à atteindre ses objectifs de justice sociale. Dans le cadre de cet article, nous ne citerons que les plus pertinents au regard de notre questionnement.

La première forme de découplage se situe dans les modalités de financement de la loi et concerne le rôle respectif et les prérogatives du niveau fédéral d'une part et du niveau des États de l'autre. Plusieurs auteur-es ont noté les ambiguïtés de NCLB dans ce domaine. Datnow et Park (2009) relèvent, par exemple, que du point de vue de sa mise en œuvre, «NCLB is a mixture of clarity and ambiguity » (p. 348).

Au niveau fédéral, Lee (2006) note qu'après la première année de mise en place, les ressources promises par la loi NCLB n'avaient pas été fournies. Dee et Jacob (2011) n'ont trouvé aucune trace d'une augmentation du financement fédéral pour l'éducation, ce qui indique que le système scolaire public a dû s'adapter à la nouvelle politique éducative sans ressource supplémentaire. Les États qui espéraient recevoir davantage que les 10\% supplémentaires prévus ont commencé à énoncer des réserves envers le gouvernement (Gamoran, 2012), car celui-ci demandait aux établissements et aux enseignant-es une charge de travail considérable pour répondre aux nouvelles attentes de NCLB sans pour autant leur donner suffisamment de budgets pour y faire face. De cette façon, il les laissait assumer le coût financier du dispositif (Foucault, 2017).

En plus de ne pas augmenter les fonds pour soutenir les réformes, le fonctionnement prévu de leur distribution n'était toujours pas en vigueur en 2009 (Montagutelli, 2009). Les instances administratives des districts percevaient encore les fonds et décidaient de leur répartition en envoyant plus souvent du matériel que de l'argent aux établissements alors que selon la loi, une enveloppe budgétaire devait aller directement à ces derniers pour qu'ils la gèrent en fonction de leurs besoins. Pour Montagutelli (2009), l'origine insatisfaisante de la distribution de ces fonds serait due à l'adaptation de la loi par les États d'une part, et au manque de coordination entre les administrations d'autre part. Selon elle, certains États se seraient appropriés la décision de la répartition du financement - jouant de leur autonomie décisionnelle et politique - dans le but d'établir " plus d'égalité entre les districts », comme le Texas qui a procédé à une refonte des allocations budgétaires vers les districts. Par ailleurs, nombre d'entre eux ont orienté ces fonds vers des dispositions de la loi, notamment vers les tests (Lee, 2006), alors qu'ils étaient initialement prévus pour le financement des programmes et pour soutenir les enfants défavorisés dans leurs apprentissages.

Ainsi, on voit que si la gestion plus souple et plus autonome n'est pas vraiment accordée au niveau local, c'est parce qu'en partie, les États régulent les fonds à leur façon et selon leurs 
intérêts. Une explication à cette gestion du financement est proposée par Lee (2006), pour qui de nombreux États avaient affirmé qu'ils se sentaient obligés de réorienter ces fonds vers des dispositions (notamment les tests) qu'ils estimaient inutiles. À ce sujet, le chercheur a observé que le gouvernement fédéral fournissait indirectement 412 millions de dollars par an pour aider à payer une partie des tests supplémentaires exigés par la loi.

Une seconde explication est proposée par Shelly (2008), pour qui de nombreux États ont résisté aux injonctions de l'État fédéral. En effet, certains États n'ont pas accepté passivement les nouvelles responsabilités qui leur ont été imposées par la loi : "Trente-huit d'entre eux ont adopté des lois en opposition à certaines dispositions de NCLB, comme le Colorado, I'Illinois, le Maine, l'Utah et la Virginie » (Shelly, 2008, p. 444).

La deuxième forme de découplage concerne la réalisation des " mesures d'ajustements ", c'est-à-dire des mesures venant sanctionner les établissements qui n'atteignent pas les objectifs fixés après plusieurs années. Ce système progressif d'interventions de NCLB ne s'est en fait pas pleinement appliqué. Certes, la transformation de certaines écoles publiques en Charter School a été effective, comme le démontre leur augmentation depuis l'entrée en vigueur de NCLB (Koppich, 2005), signifiant que les États ont probablement utilisé cette sanction comme réponse aux échecs répétitifs de certaines d'entre elles (Montagutelli, 2009). Cependant, la possibilité de remplacer toute une équipe éducative jugée défaillante ou encore de fermer un établissement n'a pas réellement été appliquée (Foucault, 2017 ; Gamoran, 2012), ni le licenciement du personnel. En effet, comme l'a relevé Koppich (2005), dans la plupart des États, il est quasiment impossible que les enseignant-es titularisé-es soient licencié-es.

Le troisième découplage que nous retenons concerne les familles et les aménagements pour les élèves en difficulté. II n'a pas pu réellement se matérialiser. En effet, lorsque le tutorat était appliqué, il n'était pas conforme aux prescriptions de NCLB et ne constituait donc pas une aide effective pour les familles. En effet, Gamoran (2012) note que les États devaient également proposer du tutorat aux élèves en difficulté dans les écoles n'ayant pas réalisé les progrès annuels escomptés (AYP) trois années de suite. Les études montrent que seuls 20\% des élèves concerné-es en bénéficient et que les effectifs des groupes de tutorat sont souvent de huit à dix élèves au lieu de un à trois. Par ailleurs, la coordination entre tuteurs/ trices et enseignant-es laisse à désirer et les compétences des tuteurs/trices ne sont ni homogènes ni réellement vérifiées (p. 17). Par ailleurs, selon Aktouf (2010), les « chèques d'éducation » (vouchers) donnés aux parents pour choisir et mettre en compétition les écoles n'étaient utilisés que dans trois états en 2010, et ce à titre expérimental et limité. Dans les autres États, le recours aux Charter Schools a été privilégié, avec l'idée qu'une gestion privée des écoles serait plus efficace et plus centrée sur l'intérêt des élèves qu'une gestion publique. Enfin, on peut aussi s'interroger sur le rôle accordé aux familles et in fine à leur implication réelle dans cette politique. À partir d'une enquête empirique auprès de 13 écoles de Seattle, Lavery (2016) s'intéresse aux connaissances qu'ont les parents des possibilités offertes par la loi NCLB. II ressort de son étude que la plupart des parents affirment être 
familiarisés avec NCLB, quel que soit le type d'école que fréquente leur enfant. En revanche, la plupart des parents ne savent pas si l'école de leur enfant figure ou non sur la liste des écoles identifiées comme devant progresser de l'État et les parents dont les enfants fréquentent ces établissements en difficulté sont encore moins susceptibles que leurs pairs d'identifier correctement leur statut scolaire. En d'autres termes, l'instrument d'empowerment des parents, notamment les plus défavorisés, se heurte à la question de la diffusion de l'information sur les écoles, ce qui limite les capacités d'action des familles.

Le quatrième découplage, et non des moindres, concerne les pratiques d'enseignement en lien avec la politique d'accountability. Dans son bilan de NCLB, Ladd (2017) s'intéresse aux conséquences pédagogiques des modalités de la loi NCLB. Elle souligne, à la suite de McMurrer (2007), le phénomène de teaching to the test : le seul fait d'évaluer le travail du corps enseignant sur la base de tests de connaissances passés par leurs élèves oriente l'enseignement dans les domaines testés au détriment d'autres domaines tout aussi légitimes sur le plan de la formation intellectuelle des élèves. En outre, écrit-elle,

NCLB a également encouragé les enseignant[-es] à restreindre les groupes d'élèves dont ils[/elles] s'occupent. Diverses études indiquent, par exemple, que l'incitation pour les enseignant[-es] à concentrer leur attention sur les élèves proches du seuil de compétence a conduit à des réductions de la réussite des élèves dans les queues de la distribution des capacités. (Ladd, 2017, p. 465)

En d'autres termes, les enseignant-es soumis-es à une forte pression du fait des règles d'accountability de NCLB agissent de façon à répondre formellement aux attentes de leur hiérarchie (la progression des élèves aux tests doit être mesurable) sans pour autant que leur action aille dans le sens d'une lutte contre les inégalités d'apprentissage. Ce découplage entre les objectifs affichés de la loi et la réalité des pratiques enseignantes donne à voir les écueils de la reddition de compte : loin de réguler les comportements d'acteurs éducatifs dans le sens des objectifs affichés de NCLB (lutter contre les inégalités d'apprentissage), la reddition de compte conduit à des actions stratégiques qui vont dans le sens d'un contournement de ces objectifs.

II s'agit là d'un effet pervers de la reddition de compte qui s'observe aussi dans la définition même des programmes. Par exemple, Sunderman et ses collaborateurs (2005, cités par Maleyko \& Gawlik, 2011) ont recueilli des données d'enquête qualitative auprès du corps enseignant de deux districts scolaires : Fresno, en Californie, et Richmond Country, en Virginie. Les enseignant-es ont déclaré avoir réduit le programme d'études en accordant plus d'attention aux domaines testés par opposition aux domaines qui ne l'étaient pas. Une enquête menée auprès de 349 districts scolaires entre 2001 et 2007 par McMurrer (2007, voir également les enquêtes nationales du Center on Education Policy33 ; Byrd-Blake, et al., 2010 ; Dee \& Jacob, 2011 ; Griffith \& Scharmann, 2008, cités par Ladd, 2017) a montré que les écoles ont augmenté le temps d'enseignement (mesuré en minutes par semaine) en 
anglais et en mathématiques de manière assez significative tout en réduisant le temps consacré aux autres matières.

Ce type de réduction des programmes a aussi été observé pour les mathématiques et la lecture, car NCLB décourageait l'utilisation de formes d'évaluation utiles à l'enseignement qui impliquent une rédaction et une analyse approfondies (Maleyko \& Gawlik, 2011). Selon ces auteur-es, certains États ont été contraints d'éliminer l'utilisation de systèmes d'évaluation sophistiqués basés sur les compétences identiques à ceux utilisés dans d'autres nations, comme les évaluations internationales PISA. Cela s'explique en partie par la forte dépendance à l'égard des tests à choix multiples, moins coûteux et plus rapides à évaluer que les questions ouvertes qui permettent de mieux tester la compréhension conceptuelle et les compétences en écriture (Ladd, 2017).

En définitive, NCLB illustre de façon exemplaire les phénomènes de découplages qui prévalent dans les politiques publiques, notamment en éducation. Ce phénomène relève d'un décalage entre la logique de la loi et celle des acteurs qui ont pour mission d'en faire la transposition dans la réalité de leurs pratiques concrètes. Qu'il s'agisse de la logique des États soucieux de leur autonomie par rapport au niveau fédéral, des obstacles institutionnels à l'application d'une version " dure » de la reddition de comptes, de l'accès à l'information des familles les plus défavorisées trop souvent démunies pour percevoir le fonctionnement de l'école ou encore de l'autonomie pédagogique des enseignant-es, tous ces phénomènes montrent - dans la perspective inaugurée par Lipsky dans son ouvrage Street level bureaucracy (1980) - que l'action publique ne prend véritablement corps et ne revêt ses véritables formes que dans son accomplissement quotidien par les acteurs eux-mêmes, dans le hic et nunc des institutions.

La question est alors de savoir quels ont été les effets de cette politique sur le niveau d'apprentissage des élèves aux États-Unis et sur les inégalités. En un mot, sur la justice sociale au sein même des processus d'apprentissage.

\section{UN SYSTÈME ÉDUCATIF PLUS JUSTE ET EFFICACE ?}

La loi NCLB a-t-elle atteint ses objectifs ? Le système éducatif étasunien est-il plus performant en 2015 qu'au début des années 2000 ? Les inégalités d'apprentissage entre élèves des minorités et élèves blanc-hes ont-elles diminué ? Qu'en est-il des inégalités liées au niveau socioéconomique des familles ? II existe une abondante littérature scientifique sur ces questions, abondance qui est liée d'une part aux données qu'a permis de recueillir la loi NCLB, et d'autre part, aux débats très vifs suscités dans la communauté académique par I'instrument de reddition de comptes.

D'abord, il faut souligner que NCLB a permis le recueil d'une masse importante de données sur le niveau d'apprentissage des élèves en mathématiques et lecture dans les différents États de l'union. Cette dimension est considérée par Ladd (2017) comme un des acquis très 
positifs de la loi, compte tenu du fait que cela permet une connaissance fine des apprentissages et des inégalités. Les chercheurs et chercheuses en sciences sociales ont pu s'en emparer pour approfondir la connaissance dans ce domaine. Ensuite, à propos de la reddition de compte, il faut souligner que cet instrument, typique des politiques néo-libérales qui se développent en éducation depuis les années 1980, a suscité beaucoup de critiques dans son principe même. On reproche non seulement à NCLB de soumettre l'éducation " à l'épreuve de la performance » pour reprendre l'expression de Maroy (2013), mais aussi la reddition de comptes dépossèderait les professionnel-les de l'enseignement de leurs savoirfaire, dévalorisés au profit d'une conception utilitaire et managériale du métier (Malet, 2009).

Notre ambition n'est pas ici de trancher ce débat sur les effets de la reddition de compte sur l'institution éducative elle-même et la profession enseignante. Plus modestement, nous tenterons dans cette troisième partie une mise en perspective des objectifs phares de la loi NCLB que l'on peut résumer par deux éléments essentiels : l'accroissement global des acquis des élèves dans des disciplines centrales et la diminution des inégalités d'apprentissage.

Pour cela, l'article de Dee et Jacob (2011) est très pertinent, car il opère une comparaison quantitative des résultats aux tests des élèves de diverses caractéristiques. II s'agit des résultats au National Assessment of Educational Progress (NAEP) dont une des caractéristiques est de permettre une comparaison des apprentissages avant la mise en place de NCLB et pendant cette mise en place. II s'agit d'évaluations qui ne dépendent pas des normes "locales » définies par chaque État, ce qui en fonde la pertinence au plan comparatif. Enfin, les données NAEP permettent une analyse fine des résultats, en fonction des performances en lecture et mathématiques. L'échantillon final des auteurs comprend entre 37 et 39 États selon la discipline et le niveau de scolarisation considéré (quatrième année et huitième année).

Au-delà de la question des effets de NCLB sur les acquis des élèves, Dee et Jacob (2011) se questionnent sur les ressorts de l'évolution constatée. Ils distinguent notamment les États dans lesquels une politique d'accountability a été mise en place dès les années 1990 c'est-à-dire bien avant la mise en place de NCLB en 2001 - et ceux pour lesquels cela n'a pas été le cas. Cela permet aux auteurs d'opérer une comparaison entre ces États et de considérer ceux dans lesquels les pratiques de reddition de compte sont anciennes comme références. Cela permet aussi aux auteurs de s'interroger sur les mécanismes qui conditionnent les effets de NCLB en lien avec les politiques de reddition de compte. Un des résultats est que les États dans lesquels des pratiques de reddition de compte n'ont pas été mises en place avant NCLB - en l'occurrence avant 1997 - ont une progression des résultats en mathématiques plus importante que dans les États où ces pratiques existaient déjà. Pour les auteurs, cela suggère que la reddition de compte a un effet positif sur les 
apprentissages des élèves, même si cet effet s'observe uniquement en mathématiques et non en lecture.

En effet, dans leur conclusion, les auteurs aboutissent à des résultats montrant des gains positifs en mathématiques, surtout pour les élèves en grade 4. En revanche, ils n'observent aucun gain significatif des dispositions de la loi NCLB en lecture. Par ailleurs, les effets de la loi sur les inégalités d'apprentissage restent limités : " [...] critics of NCLB can point to the lack of similarly robust effects on reading and the reform's limited contributions to reducing achievement gaps » (Dee \& Jacob, 2011, p. 442).

Ullmo (2009) arrive à d'autres conclusions en se basant sur les résultats des élèves aux tests de I'OCDE (PISA, 2006). Pour la chercheuse française, il y a un progrès notable en lecture depuis l'entrée en vigueur de la loi, mais les écarts se sont creusés entre les blanc-hes (560 points), les noir-es (503) et les hispaniques (518). Nous pouvons expliquer cette différence de résultats entre les données NAEP et PISA par le fait que les deux enquêtes ne mesurent pas les mêmes apprentissages. NAEP est fondée sur la mesure de savoirs, en lien avec les programmes scolaires. PISA, par la nécessité de comparer des élèves scolarisé-es dans des pays dont les programmes scolaires sont très différents, mesure des compétences. On trouvera dans Felouzis et Charmillot (2012) des éléments plus approfondis sur ce point.

II reste que, en termes de résultats de NCLB concernant les apprentissages des élèves et les inégalités, les objectifs de la politique ne sont que très partiellement atteints. Le consensus sur ce constat dans la littérature scientifique est réel même si cela ne doit pas laisser penser qu'aucune conséquence positive n'est à mettre au crédit de cette loi. Parmi ces conséquences positives, on peut citer l'obligation pour les États de recruter des enseignantes diplômé-es de l'université et compétent-es dans la matière enseignée. Par ailleurs sur le plan des programmes, Gamoran (2012) souligne que «la hausse du niveau d'exigence imposé par la loi NCLB a visiblement incité les enseignants et les chefs d'établissement à expérimenter de nouvelles méthodes pour accroître la réussite de leurs élèves » (p. 15). Enfin, la mesure des inégalités d'apprentissage en fonction des groupes ethno-raciaux et socioéconomiques a permis de mettre mieux en lumière les inégalités entre groupes démographiques (Gamoran, 2012).

\section{DISCUSSION : “A PRESSURE WITHOUT SUPPORT »?}

L'analyse de la littérature scientifique sur la loi NCLB nous conduit à formuler plusieurs constats concernant l'objet de cet article : à quelles conditions une politique éducative peutelle être juste, c'est-à-dire équitable dans son fonctionnement et égalitaire dans ses résultats ? Une reformulation plus précise de cette question pourrait être : une politique basée sur la reddition de compte, la responsabilité directe des établissements et du corps 
enseignant quant au niveau d'apprentissage des élèves peut-elle atteindre ses objectifs de limiter les inégalités d'apprentissage entre groupes sociaux et à quelles conditions?

Pour répondre à cette question, nous avons raisonné sur la dimension « mise en œuvre " de NCLB, dans la perspective de montrer qu'une loi se définit in fine plus par ses modalités concrètes de réalisation que par les principes abstraits dont elle se réclame et les visées qu'elle construit. De ce point de vue, les instruments utilisés par NCLB - normalisation par l'État fédéral, autonomie des États et des écoles, empowerment des familles et accountability - ont été examinés à l'aune de leur mise en œuvre concrète et de leurs effets sur les processus éducatifs. Dans ce cadre, NCLB est apparue comme un cas d'école. Au regard de la complexité et de la multiplicité des acteurs intervenant dans le domaine de l'éducation aux États-Unis, il est clair que le découplage entre les obligations fixées par la loi et leur transposition sur le terrain éducatif est patent, attesté par de nombreux travaux mobilisés dans cette contribution. Ce découplage s'est manifesté tant sur les plans fiscal et du financement, que sur le plan de la mise en place des mesures d'ajustement pour les établissements n'atteignant pas les objectifs, ou encore au niveau de l'empowerment des familles et à celui, déterminant, des pratiques d'enseignement.

II est toutefois possible, dans le cadre de cette discussion, d'aller plus loin dans l'analyse, car la mise en œuvre d'une politique est aussi une question de construction du sens (Datnow \& Park, 2012 ; Lessard \& Carpentier, 2015). Dans le cas de NCLB, et pour reprendre les analyses de Montagutelli (2009), Gamoran (2012) et Ladd (2017), cette construction de sens s'organise autour de l'idée de responsabilité directe des établissements et du corps enseignant dans les difficultés d'apprentissage de leurs élèves. Ces travaux ont souligné l'incomplétude de cette vision des processus éducatifs qui fait porter aux seuls acteurs éducatifs la responsabilité de l'échec scolaire et des inégalités d'apprentissage. La question de la « responsabilité » des écoles dans la progression scolaire des élèves relève pour Ladd (2017) d'une conception " étroite » des objectifs de l'éducation et des processus d'apprentissage. Si les établissements et les enseignant-es ont un rôle moteur dans les apprentissages, on ne peut leur imputer l'ensemble des processus à l'œuvre. Ceci a pu être démontré par l'analyse des caractéristiques des établissements en échec selon les critères de NCLB. Plusieurs chercheurs/euses, cité-es par Maleyko et Gawlik, (2011), ont observé que les écoles pauvres et scolarisant une proportion importante d'élèves des minorités étaient les premières à être sanctionnées par les dispositifs de NCLB (Mathis, 2004 ; Meier \& Wood, 2004 ; Sunderman, et al., 2005). Cela montre qu'une politique de justice distributive ne peut ignorer le poids des conditions d'enseignement sur les apprentissages des élèves. Ces conditions sont déterminantes pour comprendre les effets de l'exercice du métier d'enseignant-e au sein même des classes. La justice sociale en éducation passe donc par une compréhension fine des mécanismes de production des inégalités, incluant les effets de contexte et de composition. C'est déjà ce que montrait Jencks (1979), et ce que montrent 
encore les travaux plus récents sur les liens entre ségrégation scolaire et inégalités d'apprentissage (Felouzis, 2020).

De plus, sachant que le financement des districts est apporté principalement par les impôts, " [les] districts déshérités ne sont pas en mesure de financer leurs écoles correctement » (Ullmo, 2009, p. 73). Dans cette situation, les écoles les plus défavorisées ont plus de probabilité de ne pas respecter les normes AYP, ce qui entraîne une baisse de leur financement pour payer les sanctions prescrites par la loi. D'autres études rejoignent ces conclusions. Porter et al. (2005) ont observé que les écoles comptant 25\% ou plus d'élèves noir-es au Kentucky sont beaucoup plus susceptibles d'échouer au AYP. Ils ont également montré que les écoles scolarisant plus d'élèves économiquement défavorisé-es sont plus susceptibles de ne pas répondre aux exigences du AYP. Les chercheurs ont aussi trouvé qu'un nombre important d'écoles (73\%) à forte population d'élèves en difficulté n'ont pas réussi les progrès annuels. En revanche, seulement 30\% des établissements en progrès ont une population d'élèves économiquement défavorisé-es.

Ce type de résultats fait dire à Ladd (2017) que NCLB a fonctionné selon un principe de "pressure without support » qui ne permet pas réellement d'aboutir à des résultats concluant faute d'une vision globale des processus éducatifs : "NCLB placed significant pressure on individual schools to raise student achievement without providing the support needed to assure that all students had an opportunity to learn to the higher standards » (p. 466).

Tout porte à croire que cette pratique de «pressure without support », qui consiste à exercer une pression forte sur les établissements sans pour autant leur donner les moyens d'atteindre les objectifs fixés, est largement inéquitable, car elle pénalise les établissements scolarisant les élèves les plus en difficulté scolaire, défavorisé-es sur le plan de leur milieu socioéconomique et de leur appartenance à un groupe minoritaire. Définis comme « en échec » plusieurs années d'affilée par les procédures de NCLB, beaucoup d'établissements défavorisés voient leur dotation budgétaire diminuer, alors même qu'une politique redistributive des moyens irait dans le sens d'une augmentation des budgets alloués à ces établissements dont les besoins sont a priori plus importants dès lors qu'ils scolarisent des élèves plus souvent en difficulté d'apprentissage. De plus, si elles en viennent à fermer, les nouvelles écoles ne sont pas assurées de mieux réussir (Ravitch, 2007, cité par Ullmo, 2009), puisqu'elles sont vouées à se confronter aux mêmes défis que leurs prédécesseures sans bénéficier davantage de ressources.

\section{CONCLUSION}

Nous pouvons conclure cette analyse des effets de NCLB sur la justice sociale à l'école en deux points. D'abord sur le plan empirique, il apparaît que cette situation de " pressure without support » conduit à mettre les établissements les plus défavorisés en échec, car ils 
ne peuvent, sauf miracle, atteindre les objectifs de la loi. D'où l'ampleur du découplage observé dans les établissements entre les attentes de la loi et les stratégies des acteurs en situation. Cela nous conduit ensuite au deuxième point de notre conclusion. En effet, notre analyse de la littérature sur la loi NCLB montre qu'une des conditions pour atteindre un objectif de justice sociale à l'école est que les principes d'équité s'exercent pour toutes les parties prenantes de l'éducation : non seulement pour les élèves et leur famille qui ont droit à une éducation de qualité, mais aussi pour les enseignant-es et les établissements qui ne peuvent être tenus pour seuls responsables de l'ensemble des faits sociaux qui conditionnent les apprentissages et les inégalités.

Le principe de différence de Rawls, évoqué en introduction de cet article pour qualifier les principes théoriques d'une politique éducative juste, montre ici son incomplétude. Car c'est aussi en termes d'empowerment et de pouvoir d'agir des enseignant-es que la question de la justice sociale en éducation se pose. Notre article montre qu'il ne suffit pas de responsabiliser et d'évaluer les enseignant-es pour que l'école soit plus juste et équitable. II est aussi nécessaire de leur donner tous les moyens d'agir dans le sens d'une juste répartition des biens éducatifs. Si l'on admet cette idée comme vraie, on peut alors tirer de notre analyse de NCLB que l'équité des résultats (pour les élèves et leurs familles) ne peut pleinement se réaliser que dans le cadre d'une équité des conditions d'enseignement et des responsabilités, pour les enseignant-es et les établissements scolaires.

\section{RÉFÉRENCES}

Aktouf, O. (2010). L'éducation et les lois du marché. Education Canada, 42(4). https:// www.edcan.ca/wp-content/uploads/EdCan-2002-v42-n4-Aktouf.pdf

Barton, P. E., \& Coley, R. J. (2010). The Black-White Achievement gap. When Progress Stopped (Policy Information Report). Princeton, NJ : Educational Testing Service. https:// www.ets.org/Media/Research/pdf/PICBWGAP.pdf

Becker, R. (2013). Editorial. Inégalité et justice dans l'éducation en Suisse. Revue Suisse des sciences de l'éducation, 35(3), 415-423. https://www.pedocs.de/volltexte/2015/10301/ pdf/SZBW_2013_3_Becker_Editorial_Inegalite_et_justice.pdf

Bell, A. L., \& Meinelt, K. A. (2011). A past, present, and future look at No Child Left Behind. Human Rights, 38(4), 11-14. http://www.jstor.org/stable/23375611

Byrd-Blake, M., Afolayan, M. O., Hunt, J. W., Fabunmi, M., Pryor, B. W., \& Leander, R. (2010). Morale of teachers in high poverty schools: A post-NCLB mixed methods analysis. Education and Urban Society, 42(4), 450-472.

https://doi.org/10.1177/0013124510362340

Coburn, C. E. (2004). Beyond Decoupling: Rethinking the Relationship Between the Institutional Environment and the Classroom. Sociology of Education, 77(3), 211-244. https://doi.org/10.1177/003804070407700302 
Crahay, M. (2012). L'école peut-elle être juste et efficace : De l'égalité des chances à l'égalité des acquis. Louvain-la-Neuve, Belgique : De Boeck Supérieur.

Datnow, A., \& Park, V. (2009). Conceptualizing policy implementation: Large-scale reform in an era of complexity. In G. Sykes, B. Schneider \& D. N. Plank (Eds.), Handbook of education policy research (1e éd., pp. 348-361). New York : Routledge.

Dee, T. S., \& Jacob, B. (2011). The Impact of No Child Left Behind on Student Achievement. Journal of Policy Analysis and Management, 30(3), 418-446. http://www.jstor.org/stable/ 23018959

Department of Education. (2003). No Child Left Behind: A Parents Guide. https://www2.ed.gov/parents/academic/involve/nclbguide/parentsguide.pdf

Dumay X., Cattonar B., Maroy C. (2013). Modalités de mise en œuvre des instruments d'évaluation externe et institutionnalisation de la régulation par les résultats dans les établissements scolaires. In Maroy, C. (Ed.), L'école à l'épreuve de la performance (pp. 123-140). Louvain-La-Neuve : De Boeck.

Felouzis G., \& Charmillot S. (2012). Les enquêtes PISA. Paris : Presses Universitaires de France.

Felouzis G., Maroy C., \& Van Zanten A. (2013). Les marchés scolaires. Sociologie d'une politique publique d'éducation. Paris : Presses Universitaires de France.

Felouzis G. (2020). Les inégalités scolaires. Paris : Presses Universitaires de France.

Foucault, M. (2017). « No child left behind »: entre espoir et déception. Administration \& Éducation, 155(3), 29-33. https://doi.org/10.3917/admed.155.0029

Gamoran, A. (2007). Standards-based reform and the poverty gap: Lessons for No Child Left Behind. Washington, DC: Brookings Institution.

Gamoran, A. (2012). Bilan et devenir de la loi No Child Left Behind aux États-Unis. Revue Française De Pédagogie, 178(1), 13-26. http://www.jstor.org/stable/41698030

Hanushek E., \& Rivkin G. (2006). School Quality and the Black-White Achievement Gap. National Bureau of Economic Research, Working Paper No. 12651.

Hassenteufel, P. (2011). Sociologie politique : I'action publique. Paris : Armand Colin.

http://doi.org/10.3917/arco.hasse.2011.01

Jencks C. (1979). L'inégalité. Paris : PUF.

Koppich, J. (2005). A Tale of Two Approaches: The AFT, the NEA, and NCLB. Peabody Journal of Education, 80(2), 137-155. http://www.jstor.org/stable/3497064

Ladd H. (2017). No Child Left Behind. A deeply flawed federal policy. Journal of Policy Analysis and Management, 36(2). http://doi.org/10.1002/pam.21978

Lahire B. (2019). Enfances de classe. Paris : Éditions du Seuil.

Lascoumes, P., \& Le Galès, P. (2005). Gouverner par les instruments. Paris : Presses de Sciences Po.

Lavery L. (2016). What parents still do not know about No Child Left Behind and why it matters. Journal of Education Policy, 31(3), 343-361. https://www.tandfonline.com/doi/full/ 10.1080/02680939.2015.1094576? scroll=top\&needAccess=true 
Lee, J. (2006). Tracking achievement gaps and assessing the impact of NCLB on the gaps: An in-depth look into national and state reading and math outcome trends. Cambridge, MA: The Civil Rights Project at Harvard University. https://files.eric.ed.gov/fulltext/ ED491807.pdf

Lessard, C., \& Carpentier, A. (2015). Politiques éducatives : La mise en cuvre. Paris : Presses Universitaires de France.

Lipsky, M. (1980). Street-Level Bureaucracy: Dilemmas of the Individual in Public Services. Politics \& Society, 10(1), 116-116. https://doi.org/10.1177/003232928001000113

Malet R. (2009). Former, reformer, transformer la main d'œuvre enseignante ? Politiques comparées et expériences croisées anglo-américaines. Sociétés contemporaines, 23(1), 91-122.

Maleyko, G., \& Gawlik, M. (2011). No Child Left Behind: What We Know and What We Need to Know. Education, 131(3), 600-624.

Maroy, C. (2013). L'école à l'épreuve de la performance : Les politiques de régulation par les résultats. Louvain-la-Neuve, Belgique : De Boeck Supérieur. http://doi.org/10..3917/ dbu.maroy.2013.01

McDonnell, L. (2009). A Political Science Perspective on Education Policy Analysis. In G. Sykes, B. Schneider, \& D. N. Plank (Eds.), Hanbook of Education Policy Research (1e éd., pp. 73-86). New York: Routledge.

McMurrer, J. (2007). Choices, Changes, and Challenges: Curriculum and Instruction in the NCLB Era. Washington, DC: Center on Education Policy.

Meuret, D. (2012). Les effets de la régulation par les résultats (accountability) sur les politiques d'éducation aux États-Unis. Éducation et sociétés, 30(2), 75-87. https://doi.org/ 10.3917/es.030.0075

Montagutelli, M. (2009). L'école américaine dans la tourmente de No Child Left Behind. Revue française d'études américaines, 119(1), 94-105. https://doi.org/10.3917/ rfea.119.0094

National Commission on Excellence in Education (1983). A Nation at Risk : The Imperative for Education Reform. http://edreform.com/wp-content/uploads/2013/02/A_Nation_At_Risk_1983.pdf

Palier, B., \& Surel, Y. (2005). Les «trois I » et l'analyse de l'État en action. Revue française de science politique, 55(1), 7-32. https://doi.org/10.3917/rfsp.551.0007

Organisation de coopération et de développement économique. (2006). PISA 2006. Volume 2 : date / données. https://www.oecd-ilibrary.org/docserver/9789264040151-en.pdf? expires=1634910890\&id=id\&accname=guest\&checksum=688EC84EF0892C7EF45BEDB 65DDAF25D

Porter, A. (2007). NCLB lessons learned : implications for reautorization. In A. Gamoran (Ed.), Standards-based reform and the poverty gap: Lessons for No Child Left Behind (pp. 45-88). Washington, DC: Brookings Institution. 
Porter, A.C., Linn, R.L., \& Trimble, C.S. (2005), The Effects of State Decisions About NCLB Adequate Yearly Progress Targets. Educational Measurement: Issues and Practice, 24(1), 32-39. https://doi.org/10.1111/j.1745-3992.2005.00021.x

Rawls, J. (1987). Théorie de la justice. Paris : Éditions du Seuil.

Shelly, B. (2008). Rebels and Their Causes: State Resistance to No Child Left Behind. Publius, 38(3), 444-468. http://www.jstor.org/stable/20184981

Ullmo, S. (2009). L'école et l'(in)égalité des chances aux États-Unis : effets pernicieux du fédéralisme ou fondement idéologique de la démocratie américaine? Revue française d'études américaines, 1(1), 67-79. http://doi.org/10.3917/rfea.119.0067

Van Zanten, A. (2009). Choisir son école. Stratégies familiales et médiations locales. Paris : PUF.

Weick, K. (1976). Educational Organizations as Loosely Coupled Systems. Administrative Science Quarterly, 21(1), 1-19. http://doi:10.2307/2391875 